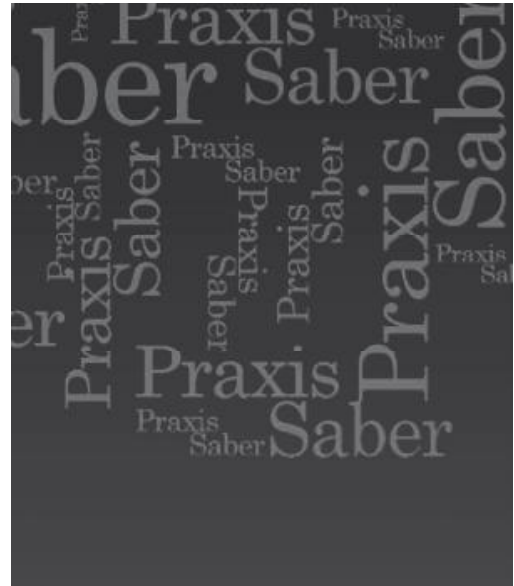

Yenny Tatiana Avellaneda

Avellaneda

Maestría en Educación,

Universidad Pedagógica y

Tecnológica de Colombia

Asesora de la Línea de Bienestar

Infantil y Juvenil, Programa

Ondas, Colciencias, Boyacá

tatiana.av.10@hotmail.com

Artículo de Investigación

Recepción: 30 de abril de 2013

Aprobación: 28 de noviembre de 2013

\section{FORMACIÓN POLÍTICA EN Y DESDE LA ESCUELA. APORTES PARA CONSTRUIR LA RELACIÓN INFANCIA-ESCUELA- POLÍTICA}

\section{Resumen}

El artículo aporta desde una mirada crítica elementos conceptuales, teóricos, metodológicos y de reflexión sobre la relación escuela-infanciapolítica; a partir de los cuales se plantea la discusión sobre el sentido de lo político en el contexto escolar. Como fundamento para ésto, se tiene en cuenta la experiencia investigativa desarrollada con comunidades escolares de la provincia de Ricaurte en Boyacá, con dos de las cuales se diseñó y desarrolló la propuesta de innovación pedagógica 'Formación política en y desde la escuela', centrada en promover la acción y la palabra como posibilitadoras de procesos de formación política en niños y niñas. Se concluye con la sistematización de la experiencia, en donde se presentan algunos elementos de análisis en torno a dos categorías emergentes, a saber: empoderamiento a partir del lenguaje y posicionamiento a través de una acción creativa transformadora.

Palabras clave: escuela, infancia, formación política, experiencia. 


\section{Political Training In And From The School. Contributions To Build The Relationship ChILdREN - SCHOOL - Politics}

\section{Abstract}

This article provides, from a critical look, conceptual, theoretical, and methodological elements of reflection on the school-children-politics relationship. Based on those elements the discussion of the meaning of politics in the school context is set out. As a basis for this, the research experience worked out with school communities in Ricaurte,Boyaca was taken into account. In two of these communities the pedagogical innovation proposal, 'Political Training in and from the School', focused on promoting action and word as enablers of political training processes in children, was designed and developed. This paper closes with the systematization of the experience, in which some elements of analysis are presented on two emerging categories, namely: empowerment through language, and positioning by means of a transformative creative action.

Key words: school, children, political training education, experience.

\section{Formation Politique À L'école Et À Partir De L'école. Apports Pour Construire La Relation Enfance-École-Politique}

\section{Résumé}

L'article apporte, d'un point de vue critique, des éléments conceptuels, théoriques, méthodologiques et de réflexion sur la relation école-enfancepolitique; à partir desquels l'on propose la discussion sur le sens de la politique dans le contexte scolaire. Sur base de ceci, on a tenu compte de l'expérience de recherche développée avec les communautés scolaires de la 
province de Ricaurte dans le Département du Boyacá; Avec deux de cellesci, a été conçue et développée la proposition d’innovation pédagogique 'Formation politique à l'école et à partir de l'école', centrée sur la favorisation de l'action et de la parole comme facilitatrice du processus de formation politique chez les garçons et les filles. On a conclu avec la systématisation de l'expérience, où se présentent certains éléments d'analyse en rapport avec les deux catégories émergentes, c'est-à-dire: autonomisation à partir du langage et positionnement au moyen d'une action créative transformatrice.

Mots clés: école, enfance, formation politique, expérience.

\section{Formação Política em e Desde a Escola. Aportes PARA CONSTRUir RELAÇÃo InFÂNCIA - Escola - Política}

\section{Resumo}

O artigo aporta desde uma mirada crítica elementos conceituais, teóricos, metodológicos e de reflexão sobre a relação escola-infância-política; a partir dos quais se faz a discussão sobre o significado do político no contexto escolar. Como fundamento para isso, se leva em conta a experiência investigativa desenvolvida com comunidades escolares da Província de Ricaurte em Boyacá, com duas das quais se elaborou e desenvolveu a proposta de inovação pedagógica "Formação política em y desde a escola", centrada em promover a ação e a palavra como fornecedoras de processos de formação política das crianças. Concluiuse com a sistematização da experiência, onde se apresentam alguns elementos de análise em volta de categorias emergentes, a saber: coragem a partir da linguagem e posicionamento a traves de uma ação criativa transformadora.

Palavras chave: escola, infância, formação política, experiência. 


\section{Introducción}

El presente artículo da cuenta de la investigación en innovación pedagógica, desarrollada durante el período 2011-2013, en el marco de la Maestría en Educación de la Universidad Pedagógica y Tecnológica de Colombia (UPTC), titulada: 'La escuela, escenario de formación política en contextos de vulneración de derechos', en la cual se plantea la pregunta ¿Qué prácticas educativas y pedagógicas posibilitan el desarrollo de procesos de formación política en y desde la escuela, en contextos de vulneración de derechos? A partir de cuya indagación, se reconocen elementos conceptuales, teóricos y metodológicos que aportan en la construcción de la relación escuela-infancia-política y dan lugar al diseño de la propuesta de innovación pedagógica 'Formación política en y desde la escuela', desarrollada con niños, niñas y maestros de dos escuelas rurales de un municipio de la provincia de Ricaurte, en Boyacá. De acuerdo con lo señalado, la experiencia se desarrolla en torno a:

- El desarrollo del proceso metodológico, enmarcado dentro de una investigación de corte cualitativo y un enfoque socio-crítico, a partir de los cuales se buscó generar un conocimiento contextualizado de acuerdo con la realidad sociocultural indagada, en un proceso que parte de la práctica y vuelve a ésta para mejorarla. Desde la dinámica de la investigación acción, se destacan entonces, como procesos significativos objeto de la sistematización, los tres ciclos del desarrollo de la propuesta pedagógica: 'Cuestionamiento e indagación desde el pensamiento infantil', 'Develamiento en torno a la vulneración de derechos' y 'Posicionamiento, o el reconocimiento de posibilidades de acción'.

- El proceso de construcción de realidad, en donde se problematizan tres aspectos de la realidad educativa, a saber: la situación de la infancia desde la cotidianidad de sus contextos escolares y comunitarios, la función que cumple la escuela en contextos de vulneración de derechos y los vínculos que se establecen al interior de la escuela.

- La discusión con los autores, donde se establece un diálogo con las posiciones teóricas de autores como Bárcena, Mélich, Freire, Arendt y Narodowski y la realidad problematizada; en el sentido de poder indagar acerca de la relación infancia, escuela y política, como categorías estructurantes que permitan construir los posibles sentidos y significados de lo político en el contexto escolar. 
- La sistematización de la experiencia, mediante la cual se tejen, releen y resignifican los tres ciclos desarrollados en la propuesta, en un ejercicio de reflexión sobre la propia práctica, que permita rescatar de ésta los aprendizajes y nuevos conocimientos construidos por los diferentes caminos transitados, de los cuales emergen como resultados, dos categorías de análisis: empoderamiento a partir del lenguaje y posicionamiento a través de una acción creativa transformadora.

\section{Diseño de investigación}

La experiencia en innovación pedagógica de la cual da cuenta este artículo, se enmarcó dentro de una investigación de corte cualitativo y un enfoque socio-crítico, a partir de los cuales se buscó generar un conocimiento contextualizado de acuerdo con la realidad sociocultural indagada; teniendo en cuenta los sentidos y significados que los actores sociales involucrados atribuyen a sus prácticas, posibilitando desde la acción y la reflexión de las mismas, la construcción de nuevas comprensiones y sentidos a partir de la experiencia. Esto orientado por un interés emancipador o liberador cuyos saberes son predeterminados en las acciones cognoscitivas o modos posibles del pensamiento, a partir de los cuales la realidad se constituye y se puede transformar (Kemmis, 1988: 143).

La dinámica de investigación que desde el enfoque socio-crítico se plantea, rompe entonces con la separación entre el sujeto y el objeto; lo cual implica que tanto el maestro-investigador como los niños y niñas se constituyen en protagonistas de la experiencia, a través de un proceso de autorreflexión y acción transformadora como parte del proceso mismo de la práctica innovadora. De esta manera, la investigación se desarrolló a partir del diseño, sentido y procedimientos aportados por la investigaciónacción, la cual, para el caso de este trabajo, permitió articular la práctica educativa con la investigación misma.

Se asume entonces la investigación-acción, en el sentido de lo planteado por Kemmis (1984), como una práctica autorreflexiva que se pone en marcha en situaciones sociales en las que se busca mejorar la racionalidad y justicia de: a) las propias prácticas sociales o educativas, b) la comprensión de estas prácticas y c) las situaciones e instituciones en que estas prácticas tienen lugar. En consecuencia, se diseña y desarrolla la propuesta de innovación pedagógica 'Formación política en y desde la escuela', centrada en el papel de la acción y la palabra como posibilitadoras de procesos de formación política en niños y niñas desde el desarrollo del pensamiento crítico. 
Se plantean así, tres ciclos a manera de espiral: 'Cuestionamiento e indagación desde el pensamiento infantil', 'Develamiento de la realidad en torno a la vulneración de derechos' y 'Posicionamiento, o el reconocimiento de posibilidades de acción'; al interior de los cuales se desarrollan estrategias y actividades desde el lenguaje y la investigación, en relación con los sentidos e intenciones propios de cada uno de estos ciclos.

\section{Contexto de la problemática}

La experiencia desarrollada como asesora del Programa Ondas, Colciencias Boyacá, en la línea de Bienestar Infantil y Juvenil, permitió el acercamiento con distintas realidades que afronta la infancia en Boyacá en relación con la problemática de vulneración de derechos. En este sentido, la experiencia desarrollada con comunidades escolares de la provincia de Ricaurte, permitió problematizar la función que cumple la escuela en estas comunidades, las relaciones entre adultos y niños y algunas prácticas educativas que limitan las posibilidades de formación de sujetos críticos, reflexivos, creativos y deliberantes.

De esta manera, se fue generando un proceso de problematización a partir del análisis de elementos de la realidad indagada, desde los cuales se plantean diversos interrogantes que buscan cuestionar aspectos de esta realidad desde una perspectiva crítica; reconociendo así, la emergencia de nuevas prácticas educativas y pedagógicas posibilitadoras de procesos de formación política en niños y niñas.

\subsection{Infancia y cotidianidad}

¿Cómo viven niños y niñas su infancia en la cotidianidad de sus contextos?

A partir del trabajo pedagógico desarrollado con niños, niñas y maestros de escuelas rurales de la provincia de Ricaurte en Boyacá, vinculados al Programa Ondas de Colciencias en la línea de Bienestar Infantil y Juvenil ${ }^{1}$, se pudieron evidenciar diferentes problemáticas que se presentan al interior de estas comunidades.

1 Línea en la que niños, niñas y jóvenes plantean sus preguntas en torno a situaciones que afecten su bienestar, y a partir de éstas, emprenden procesos de indagación en sus contextos escolares y comunitarios, en compañía de sus maestros y del asesor de línea. 
Así por ejemplo, se encontró que como único medio de sobrevivencia, la mayoría de las familias de los niños y niñas, trabajan como jornaleros en los trapiches, moliendo y procesando caña de azúcar en el caso de los hombres, y en el caso de las mujeres, empleándose en diversos oficios como cocinar, limpiar, cortar caña; en arduas y extenuantes jornadas laborales que les impide tener una mayor vinculación con sus hijos e hijas, lo cual ha llevado a generar situaciones de abandono para los niños y niñas.

Según lo relatado por las maestras, en la mayoría de los casos, la mamá se ha ido o el papá o ambos y los pocos niños y niñas que viven con sus padres, después de salir de la escuela permanecen solos porque sus padres trabajan todo el día, de manera que al decir de la profesora "los niños están abandonados, sin nadie que los oriente?

$\mathrm{Al}$ respecto, se identificaron varios casos en los cuales los niños y las niñas ni siquiera viven con alguno de sus progenitores, sino que están a cargo de sus abuelos, tíos u otros familiares, o incluso de algún vecino. En palabras de los niños y niñas estas situaciones fueron problematizadas de la siguiente manera:

¿Por qué los padres dejan abandonados a sus hijos?

¿Por qué los niños y las niñas son maltratados por sus padres y demás familiares?

¿Cuáles son las razones porque nuestros padres nos abandonan [sic]? ¿Es buena idea cambiar a mis padres? ?3 $^{3}$

Vinculado al fenómeno de violencia y abandono por parte de los padres, se encuentra que también existe una escasa cultura de apropiación y protección de los derechos de niños, niñas y mujeres en estos contextos. Lo cual se evidencia en el trato que reciben los niños y niñas por parte de los adultos y el trato que reciben las mujeres por parte de los hombres. Frente a lo cual, los maestros de las escuelas refieren que: "es algo que siempre ha existido aqui (en el municipio), yo desde que me acuerdo han pasado cosas así", "ya uno a veces ni se extraña".

2 Tomado del diario de campo del investigador, a partir de los conversatorios y diálogos informales con los maestros.

3 Tomado de los diarios de campo de los niños y niñas.

4 Tomado del diario de campo del investigador, a partir de los conversatorios y diálogos informales con los maestros. 
Situaciones que han llevado a que niños y niñas asuman actitudes pasivas frente a estas problemáticas sociales, haciendo de la violencia, el abandono y la vulneración de derechos una experiencia permanente y naturalizada, en la que para ellos no hay más salida que acogerse a la protección y el amparo del adulto, quien de manera contradictoria actúa al mismo tiempo como maltratador y protector.

El hecho de que sea el adulto el responsable de proteger, sancionar y restablecer los derechos, ha llevado a que niños y niñas asuman una mirada externa de sus derechos, en términos de un "regalo", "favor", "don" los adultos (a través del Estado) hacia ellos, viéndose condicionada de esta manera, su vivencia de la ciudadanía, al estar supeditada a la actuación del adulto.

Frente a estas situaciones tan conflictivas y adversas, los niños y niñas asumen pensamientos y actuaciones que reflejan impotencia para asumir la realidad a partir de otras posibilidades. Para ellos, estas situaciones de abandono, indiferencia, negación, violencia, resultan incuestionables, no les sorprende que sucedan y no imaginan que puedan ser de otra manera. Tal como lo manifiesta Xiomara con la expresión "el mundo es así profe, qué le vamos a hacer", con la cual responde ante el cuestionamiento que se le hace de su comportamiento luego de haberle pegado a otro niño de menos edad que ella.

\subsection{El lugar de la escuela en la comunidad}

¿Cómo se moviliza la escuela como espacio de formación en contextos de vulneración de derechos?

"La escuela es aburrida porque nunca pasa nada"

(Paula, niña de 9 años).

En relación con las problemáticas presentadas en el contexto, es notoria la escasa incidencia que tienen las escuelas en sus comunidades, tornándose pasivas, inmóviles y escasas de propuestas ante tales situaciones. En la práctica se ve cómo estas escuelas terminan reduciendo su acción

5 Estas son algunas de las respuestas dadas por los niños y las niñas a la pregunta ¿Qué son para ti los derechos? Formulada en la encuesta de Nacho Derecho. 
exclusivamente a difundir conocimientos, modelar comportamientos y crear hábitos; desconociendo aspectos sociales y culturales que están incidiendo fuertemente en el aprendizaje y comportamiento de los estudiantes, así como en el desarrollo de las prácticas educativas.

En este sentido, si bien los niños, niñas, maestros, padres de familia y comunidad en general, tienen conocimiento de estas situaciones de vulneración de derechos, sus acciones e iniciativas para enfrentar estas problemáticas son casi inexistentes, y las pocas que se dan son de orden sancionatorio y/o normativo; sin que medien procesos formativos que permitan la formación de un sujeto reflexivo y crítico.

Por tal razón, los conocimientos y comportamientos promovidos por la escuela resultan descontextualizados, impuestos y carentes de sentido para los niños y niñas, quienes asumen la escuela como una obligación y no reconocen en la educación ninguna posibilidad de transformación para sus vidas. Encontrando como única novedad el juego y la socialización con sus pares; sin embargo, estas son prácticas muy limitadas para los niños y niñas debido a los pocos espacios que la escuela les brinda. En este sentido, resultan muy ilustrativas las palabras de los niños y las niñas ${ }^{6}$ :

Juan se demoró bastante en representar lo que más le gustaba de su escuela. Al preguntarle ¿qué es lo que a ti más te gusta de tu escuela?, se quedó pensando pero no escribía ni dibujaba nada, entonces volví a preguntarle ¿Qué es lo que más te divierte o te gusta de venir a la escuela?, volvió a quedarse pensativo y luego de un rato realizó el dibujo. Le pregunte: ¿abi qué estás haciendo? y escribió "jugamos trompo". Frente a la pregunta: ¿Qué es lo que más te gusta de tu escuela? Paula con una gran sonrisa manifiesta "lo más divertido de la escuela son mis amigos".

En relación con los maestros, se les siente cansados, desmotivados, pero también muy preocupados, tanto por las situaciones que viven los niños como por su mismo rol de maestros en estos contextos. A pesar de que todos los maestros tienen conocimiento y vivencian a diario cada una de estas problemáticas, sus prácticas no se piensan a la luz de estas situaciones. Por el contrario, presentan muy poca iniciativa e interés por cuestionar, replantear y resignificar sus prácticas a la luz de estas necesidades que presenta el contexto y que la educación no puede pasar por alto.

6 Registro de entrevistas a grupos focales. 
"Después de ver la película, los niños salieron a recreo y yo aproveché para hablar con la profesora, quien se mostró muy preocupada por los problemas que se han venido acrecentando en relación con la convivencia en la escuela y la vida familiar de los niños y niñas. Al respecto me habló puntualmente de varios casos frente a los cuales ella se siente muy desesperanzada [...]"

En tal sentido, la propuesta educativa de las escuelas resulta débil frente a las situaciones a las que deben hacer frente a diario tanto niños y niñas como maestros; la mayoría de los cuales, ya han asumido estas situaciones de vulneración de derechos como algo natural de sus contextos, ya que la mayoría de ellos son oriundos de la región y han crecido, según lo relatado por ellos mismos, en medio de estas mismas condiciones, lo cual dificulta que puedan tener una mirada crítica frente a lo que viene sucediendo y tomar consciencia de la manera como sus prácticas, en algunos casos, actúan como reproductoras de este orden social.

En las prácticas educativas se reconoce una escasa participación de los niños y las niñas en la vida escolar, lo cual hace que presenten un gran desinterés por las clases, situación que se encuentra relacionada con el desarrollo de prácticas educativas autoritarias, desde las cuales se asume el conocimiento como algo ya instaurado, que se debe memorizar, que no admite ningún cuestionamiento por parte de los niños y las niñas. Por tal razón, la relación de los niños y las niñas con el conocimiento, es una relación pasiva, aburrida y sin sentido.

Dayana considera que lo más aburrido de la escuela son las clases de naturales: "no entiendo nada", ¿y por qué no entiendes nada? "porque la profesora solo nos regaña".

\subsection{Formas de vinculación en el contexto escolar}

¿Quéformas de vinculación establecen los niños con los adultos y con sus pares?

Luego, a cada uno de los estudiantes, le di el nombre de un niño de la escuela del Salvial para que le escribieran una carta contándole sobre ellos y sobre su escuela. Todos se animaron mucho con la actividad y preguntaban cómo escribirla; entonces la profesora les hizo una muestra en el tablero (diario de campo del investigador).

7 Diario de campo del investigador. 
A partir de la interacción con los maestros, estudiantes, padres de familia y personas de la comunidad, se evidencian y cuestionan las manifestaciones de poder que se ejercen en las relaciones entre adultos y niños y entre los mismos niños con sus pares, las cuales circulan asociadas a mecanismos de control, restricción y subordinación, limitando la posibilidad de participación de los niños y las niñas en sus contextos de actuación.

\section{La profesora pasa constantemente por el salón para ver que los niños estén trabajando; les llama la atención por estar fuera de sus puestos y se queda un rato observándolos desde la puerta, pero cuando la profesora se vuelve a ir, los niños nuevamente se levantan de sus puestos $^{8}$.}

Frente a este tipo de prácticas algunos niños y niñas responden de manera pasiva, sometiendo sus voluntades, deseos e intereses al poder del maestro, quien termina decidiendo por ellos. Ante esta situación los niños se plantean interrogantes como: "¿Por quélos profesores dejan a los niños sin recreo?” Lo cual ha llevado a que estos niños y niñas se sientan completamente dependientes del maestro y recurran constantemente a él para que les diga qué hacer. En el extremo opuesto se encuentran los niños y niñas que se resisten de diferentes formas al poder del maestro, desacatando sus órdenes, rechazando su voluntad, negándose a ser lo que ellos quieren que sean. Son los niños catalogados como indisciplinados por sus profesores porque no se someten a su voluntad. En este caso los niños y niñas se cuestionan aspectos como:

\section{“iPor qué los niños ensultan [sic] a los profesores"}

Asimismo, se pudo observar que las prácticas de sometimiento del otro, tanto en los adultos como en los niños y niñas, se usan en la mayoría de los casos en beneficio personal, para lograr intereses particulares por encima de los intereses de los demás. De ahí surgió la pregunta por el lugar y el sentido de lo político en el contexto de la escuela, y por las prácticas que posibilitarían el desarrollo de una propuesta de formación política surgida en y desde la escuela.

Frente a esta problemática surgen otros tantos interrogantes, a partir de los cuales se entra en diálogo con los autores, para construir las condiciones de posibilidad de una propuesta de innovación pedagógica, planteando la

8 Diario de campo del investigador. 
discusión y reflexión en torno a: ¿Qué prácticas educativas y pedagógicas posibilitan el desarrollo de procesos de formación política en y desde la escuela?

\section{En diálogo con la teoría. Aportes de los autores para avivar la discusión.}

Los diversos cuestionamientos que se fueron generando en el proceso de construcción de realidad, suscitaron el interés y la necesidad de indagar por referentes acerca de la infancia, la escuela y la política como categorías estructurantes que permitieran construir los posibles sentidos y significados de lo político en el contexto escolar, lo cual permitió identificar y analizar diferentes posturas teóricas; las cuales se ponen en diálogo a continuación con los aspectos de la realidad anteriormente problematizados.

\subsection{De la infancia moderna a las infancias en constitución}

La infancia como construcción histórica, da cuenta del proceso de reconocimiento de niños y niñas como actores sociales, ocurrido entre el siglo XVII y el siglo XX; durante los cuales tuvieron lugar profundas transformaciones tanto en los sentimientos y las creencias, como en las prácticas y discursos relacionados con la infancia. La construcción de la categoría moderna de infancia marca así una importante diferenciación entre niños, niñas y adultos como actores sociales; al mismo tiempo que determina entre ellos una manera de relacionarse, hecho que se considera de gran trascendencia y con grandes consecuencias tanto a nivel social, político y educativo.

En la modernidad comienza a despertarse un interés por el desarrollo del niño (a) que se evidencia en la aparición de diferentes discursos que tienen como objeto el estudio del cuerpo infantil, al tiempo que se desarrollan de manera paralela las primeras ideas sobre la escolarización. Es así como escuela e infancia se constituyen y afectan recíprocamente como categorías centrales del proyecto de la modernidad. En este sentido Narodowski afirma que:

El nacimiento de este nuevo sentimiento respecto de la infancia - de nuestro sentimiento moderno a este respecto- constituye el síntoma de una profunda mutación en la cultura occidental; de una transformación en las creencias y prácticas en las que la producción de discurso pedagógico va a ocupar [...] un significativo lugar tanto 
en la producción de la transformación como en el plano de las consecuencias que ésta acarrea (1994: 33).

Este nuevo interés por la infancia que Ariés describe como "sentimiento de infancia", se configura en la modernidad dando lugar a nueva idea del niño (a) como sujeto inacabado, carente y necesitado, y con un nuevo estatus social. "Ahora la infancia es individualizada a partir de un proceso lento de demarcación y reinserción de otro modo en la sociedad" (Narodowski, p. 31) que dará lugar a la construcción de un nuevo rol social para los niños y niñas.

Estas transformaciones traen consigo la aparición en la sociedad, del cuerpo infantil, y de su concepción de cuerpo que ha de ser cuidado, protegido, amado y educado; constituyéndose así el amor, la protección y la educación como valores y pilares de la escuela y la familia, transformándose radicalmente la actitud y la relación de los adultos con los niños y las niñas. Relación que en lo sucesivo estará marcada por la aparición de la dependencia como nuevo rasgo característico de la infancia. Según Martínez :

Es una constante en estudios y tratados sobre la conservación de los niños, que éstos aparezcan invariablemente caracterizados, además de incompletos, como dúctiles y maleables; es decir, como seres en edad más que propicia para la instrucción [...] esa necesidad de completar a los menores, de rectificar sus carencias físicas y espirituales, señaló también a la escuela como el lugar y el tiempo propicio para la normalización de la población a través de la escolarización (2011: 51).

La producción de los discursos pedagógicos propios de la modernidad dará cuenta entonces de niños y niñas convertidos en alumnos, no adultos en camino de serlo, cuya principal carencia estará marcada por la falta de razón (adulta), con la cual se justifica la acción educativa del adulto hacia el niño. "Esta falta de razón adulta como ingrediente central de la infancia, ineludiblemente desemboca en la necesidad de una protección específica ya que el niño es incapaz de comportarse en forma autónoma: su ley es la ley del adulto" (Narodowski, 1994: 38). Lo cual tendrá en adelante profundas incidencias en las formas de relación entre adultos, niños y niñas. En adelante estas relaciones estarán marcadas por una fuerte desigualdad y asimetría, establecidas por las características otorgadas a cada uno de ellos. 
Frente a las ideas del niño (a) como sujeto dependiente, desvalido, indefenso, regulado, clasificado y objeto de control, impulsadas por el pensamiento de la modernidad, se propone pensar una visión mucho más amplia que permita reconocer el carácter contextual, vincular, histórico y cultural de la subjetividad ${ }^{9}$. Por consiguiente, se tendría que hablar ya no de una infancia sino de diversas infancias que se constituyen a partir de la experiencia.

Al respecto, y siguiendo a Narodowski (1999) se pueden distinguir en la realidad cotidiana de los niños y niñas, al menos dos tipos de infancias que ponen en crisis la idea de la infancia en su sentido moderno. Dos puntos de fuga que diluyen esa imagen del niño (a) obediente, heterónomo y tierno, que aún hoy se mantiene presente en muchas de las prácticas escolares.

La infancia hiperrealizada. Es la infancia que se construye a partir de la realidad virtual y que debido al acceso que tienen de medios e información dejan de ocupar el lugar del no saber. Es la infancia de la inmediatez, del consumo, de la velocidad, del cambio, de la cultura tecnológica y digital.

Infancias desrealizadas. Es la infancia que se construye a partir de la realidad real de sus contextos (pobreza, violencia, desigualdad). Son los niños ( $y$ niñas que se escapan de la infantilización; que se construyen desde la necesidad de autonomía e independencia que necesitan para sobrevivir en estos contextos, creando así, sus propias categorías morales. Se trata de infancias excluidas, marginadas, desrealizadas.

\section{De la escuela disciplinaria, a la escuela como contexto de formación política}

En el intento de tratar de comprender las dinámicas propias sobre las que funciona y se mantiene hasta nuestros días la escuela disciplinaria, es necesario analizar su relación con el proyecto de la modernidad, pues éste creó un mundo basado en estructuras sociales masculinas, racionales, adultocéntricas y eurocéntricas, a partir de las cuales se clasificaron y jerarquizaron las culturas, haciendo una distinción entre dominantes y

9 De acuerdo con Torres "La categoría de subjetividad nos remite a un conjunto de instancias y procesos de producción de sentido, a través de los cuales los individuos y los colectivos sociales construyen y actúan sobre la realidad, a la vez que son construidos como tales” (2000: 8). 
dominados; excluyendo así, todas aquellas manifestaciones culturales que se alejaran del referente impuesto, tales como la infancia, el universo de lo femenino y las culturas populares.

En este sentido, la escuela como institución de la modernidad, moviliza a través de sus prácticas un orden social, cultural y educativo que privilegia, jerarquiza, disciplina, fragmenta y desvincula saberes y culturas; creando y reproduciendo situaciones de dominación, desigualdad, injusticia y exclusión que es necesario analizar desde una mirada crítica que permita develar el funcionamiento de la estructura educativa moderna basada en presupuestos y promesas de organización y progreso, para poder así analizar y comprender cómo funciona y qué dinámicas sociales crea y recrea.

Como una de sus mayores funciones, la escuela de la modernidad tiene a su cargo el disciplinamiento, que con el desarrollo del capitalismo se combina con las prácticas de control, las cuales también se sostienen sobre la idea de dominación del otro; el disciplinamiento a través de técnicas que buscan gobernar y modelar el cuerpo, imponiéndole una identidad y el control a través de técnicas de regulación y administración de los comportamientos (Cubides, 2006).

El mantenimiento de estas dinámicas de dominación, reflejadas en las prácticas cotidianas de la escuela, no da lugar al desarrollo de procesos de formación que logren afectar las historias de vida de niños y niñas, quienes manifiestan sentimientos de aburrimiento, desasosiego, frustración, cansancio y resistencia frente a estas prácticas. Lo cual evidencia la actual crisis de sentido que atraviesa la escuela, crisis que no le permite constituirse como referente de actuación para niños y niñas quienes se ven enfrentados diariamente a dos realidades: el mundo controlado que representa para ellos la escuela y el mundo cada vez más incierto en el que vivimos.

Ante estas realidades que enfrenta actualmente la escuela, se puede decir que esta institución, como impulsora del proyecto de la modernidad, entra en crisis; se cuestiona entonces su función en la sociedad, su carácter disciplinar, su estructura jerárquica y excluyente. Como alternativa a la escuela disciplinar, se propone entonces la escuela como contexto de formación política. Destacando que, como espacio de formación política y no como un simple lugar de instrucción, la escuela cumple un importante 
papel en la vida de niños, niñas y sus comunidades. Al respecto sostiene Freire:

Hay otra tarea que cumplir en la escuela a pesar del poder dominante y por causa de él — la de desopacar la realidad nublada por la ideología dominante. Obviamente, ésta es la tarea de los profesores y profesoras progresistas que saben que tienen el deber de enseñar en forma competente los contenidos pero que saben también que al hacerlos se obligan a desvelar el mundo de la opresión. Ni contenido solamente, ni desvelamiento solamente — como si fuera posible separarlos - sino el desvelamiento del mundo opresor por medio de la enseñanza de los contenidos (1999: 59).

De acuerdo con Freire, la dimensión política de la escuela conlleva a que su compromiso y responsabilidad con las comunidades trascienda la enseñanza de contenidos, normas y procedimientos para constituirse en un referente importante de sus identidades, en la medida en que los procesos de formación despliegan procesos de construcción de subjetividad.

En Freire se reconoce que una de las funciones relacionadas con la dimensión sociocultural y ética de la escuela es la relacionada con la formación política, campo desde donde es posible potenciar conocimientos, actitudes, valores, que permitan el desarrollo de los estudiantes como sujetos con posibilidades de pensar, actuar e incidir en la transformación de su realidad cotidiana a través de la participación en sus contextos de actuación.

La escuela como espacio de formación política les ofrece a estudiantes y maestros la oportunidad de encontrarse en experiencias que trascienden la lógica disciplinar y de control que tiene lugar en los procesos de instrucción, los cuales funcionan bajo la idea de la existencia de un sujeto (niño (a)/estudiante) vacío, carente dependiente e inferior que justifica la necesitad de otro sujeto (adulto/maestro) que detenta frente a aquel una condición de poder.

\subsection{La política como acción creativa y vinculante}

"La politica surge en el entre y se establece como relación" (Arendt, 2001: 46)

En la discusión sobre el sentido de lo político, se destaca la posición teórica de Hannah Arendt (2001), para quien el sentido de la política atraviesa 
la acción humana y se reconoce desde la posibilidad de aparecer, nacer, visibilizarse en el espacio público, como espacio común. En Arendt, la política encuentra su esencia en la natalidad, entendida como capacidad de creación; reconociendo así la posibilidad del ser humano para recrear el mundo a través de la acción y la palabra.

Para Arendt la palabra hace posible tener una experiencia de mundo común, "sólo hablando es posible comprender, desde todas las posiciones, cómo es realmente el mundo. El mundo es pues, lo que está entre nosotros, lo que nos separa y nos une" (2001:19). Se reconoce a partir de estos planteamientos, que la política tiene su significado en el estar juntos desde la pluralidad como realidad y necesidad ineludible para la vida de los seres humanos.

En Arendt el concepto de política se basa en la existencia básica de dos condiciones humanas: la acción y la pluralidad. La acción en el sujeto hace referencia a la lucha, a la iniciativa y a la creación que comparte con otros; es la acción la que posibilita el lenguaje entre iguales en un mundo común, como lugar de lo público y lo político. Es por eso que lo político se expresa en la acción, diferente de la labor y del trabajo. En este sentido, "la politica trata del estar juntos y los unos de los otros de los diversos" (2001: 46).

\subsection{El papel de lo político desde las pedagogías críticas}

Las pedagogías críticas buscan el fortalecimiento de lo político a través del reconocimiento de las distintas subjetividades que circulan dentro y alrededor de la escuela, entendida como escenario de formación de sujetos desde una perspectiva crítica. De esta manera, plantean que tanto maestros como estudiantes tienen una responsabilidad ética y política con su contexto.

Proponen entonces, la práctica educativa entendida como práctica política, perspectiva que reconoce el poder, la diferencia y la desigualdad, asumiendo lo político desde los discursos y acciones vinculantes entre los sujetos. De esta forma, se plantea lo político como un actuar con los otros a través del reconocimiento de las diferencias que nos unen y nos separan como miembros de un mundo en común; problematizando así, relaciones de poder y subordinación. 
En este mismo sentido de lo político construido y expresado en el lenguaje, Bárcena y Mélich (2000) siguiendo a Arendt, señalan que el ser humano vive en un mundo de signos, los cuales son susceptibles de interpretación y narración. Teniendo en cuenta ésto, consideran que la construcción de la realidad política es una construcción de orden simbólico, en cuanto afirman que "lo simbólico es ese gesto que remite siempre a un sentido que no se encuentra claramente visible de modo inmediato" (2000: 50). Para estos autores, lo político es una construcción colectiva que se configura a través del lenguaje.

Entre tanto Freire plantea como fin de la educación la participación y transformación como aspectos importantes en la configuración del sujeto político, ya que considera que la capacidad de intervenir y decidir en ciertos niveles de poder hace parte del derecho de ciudadanía, mediante el cual se reconoce la voz y la actuación de los sujetos en las instancias de poder. Al respecto indica que:

Es preciso, democratizar el poder, reconocer el derecho natural de voz a los alumnos, a las profesoras, reducir el poder personal de las directoras, crear nuevas instancias de poder con los consejos de escuela, deliberativos y no solo consultivos, a través de los cuales, en un primer momento, padres y madres vayan teniendo injerencia en los destinos de la escuela de sus hijos, y en un segundo momento esperamos que la propia comunidad local teniendo a la escuela como algo suyo, se haga presente en la conducción política educacional de la escuela (1999: 83).

Para Freire, la participación se constituye en un aspecto propio del ejercicio ciudadano, que busca descentralizar el poder y reconocer que todos los sujetos son capaces de actuar de forma política, entendiendo la política como capacidad propia del ser humano desde sus dimensiones como ser social e histórico y por tanto con posibilidades, en este sentido, se entiende que lo político no puede restringirse solamente a ciertos actores legitimados para ello.

En Freire lo político implica asumir posturas frente a la realidad, tomar decisiones, hacer rupturas y asumir riesgos, en el sentido de vivir la historia como posibilidad y no como determinación, asumiendo la respectiva libertad que ello implica y exige. Con lo cual, el sujeto como ser inacabado, se va construyendo poco a poco en la práctica en la cual toma parte. 


\section{Sistematización de la experiencia 'Formación política en y desde la escuela'}

La sistematización de la experiencia, se desarrolló a través de la reconstrucción de los ciclos, a partir de los cuales, se reflexiona y analiza la experiencia en torno a dos categorías emergentes: empoderamiento a partir del lenguaje y posicionamiento a través de una acción creativa transformadora.

Durante el primer ciclo puesto en marcha 'cuestionamiento $\boldsymbol{e}$ indagación desde el pensamiento infantil', se asumieron como referentes importantes para el desarrollo de la propuesta, los procesos de indagación basados en la investigación como estrategia pedagógica, creada y promovida por el Programa Ondas de Colciencias. De esta manera, se buscó que a través del trabajo pedagógico con la pregunta, se propiciaran procesos de indagación sobre los derechos de los niños y las niñas, a partir de preguntas formuladas por ellos mismos.

Empoderamiento a partir del lenguaje: Las preguntas formuladas posibilitaron la movilización de los saberes de los niños y niñas en busca de nuevos lugares de saber y experiencia, a través de los cuales se retroalimentaron, cuestionaron y superaron algunas visiones impuestas de la realidad. El desarrollo de estas preguntas a través de la indagación, dio lugar a la problematización de algunos sentidos y significados en torno a prácticas y discursos cotidianos en relación con los derechos de los niños, aportando elementos de análisis y reflexión sobre la realidad cuestionada por la pregunta.

Asimismo, el proceso de construcción y reconstrucción permanente de sus propias preguntas, les permitió a los niños y niñas, reconocerse como sujetos de saber, de curiosidad, asombrarse y cuestionarse por las situaciones que acontecen a diario en sus contextos; posibilitando en ellos nuevas formas de vinculación con el conocimiento, con el maestro, con el entorno, con el mundo de la experiencia, asumir un rol más activo, participativo y visible en la escuela y transformar su visión de la realidad por un mirada mucho más crítica.

Posicionamiento a través de una acción creativa transformadora: Desde la experiencia desarrollada con la pregunta, se reconoce en los niños y niñas una gran preocupación por el mundo, una enorme capacidad 
de extrañamiento frente a situaciones de su vida cotidiana en contextos de vulneración de derechos y un interés creciente por transformar estas situaciones. La pregunta en los niños (as) se entiende entonces, como una manera de posicionarse frente al mundo con asombro y deseos de reconstruirlo.

En el desarrollo del segundo ciclo, "Develamiento de la realidad en torno a la vulneración de derechos”, la indagación de las preguntas problematizadoras y la construcción de realidad que se logra a partir de ésta, dan lugar a procesos de reflexión y análisis mediante los cuales se busca construir nuevos sentidos y significados de la realidad, que permitan desentrañar y develar ciertos aspectos de ésta, para deconstruirlos y resignificarlos desde la mirada de los niños y niñas. De esta manera, las acciones propuestas en este ciclo buscaron desarrollar en los niños (as) una actitud autoreflexiva en relación con sus historias de vida, que a partir del relato de lo vivido, les permitiera entrar en diálogo con el mundo a partir de su experiencia.

Empoderamiento a partir del lenguaje: a través de la discusión colectiva con los niños y las niñas, se promovieron procesos de problematización y reflexión sobre las situaciones de vulneración presentadas en sus contextos; procesos desde los cuales fue posible generar el desocultamiento de situaciones de abandono, indiferencia y negación de los niños y niñas en sus contextos escolares y familiares. Visibilizando así, formas de poder, de control, imposición y sometimiento en las relaciones entre adultos y niños y niñas, naturalizadas en las prácticas cotidianas.

\section{Posicionamiento a través de una acción creativa transformadora:} Los espacios de discusión colectiva promovidos en este ciclo, se desarrollaron a través de acciones como la lectura y escritura de cuentos, las cuales permitieron reconocer y darle un lugar a las historias de los niños y niñas, contadas a través de su propia voz. Pasando de un desinterés abiertamente manifiesto por leer y escribir, a una necesidad de expresarse, imaginar, recrear y visibilizarse a partir de sus narraciones. En este sentido, resultó muy emocionante la experiencia de sentir cómo los niños y las niñas a partir de la identificación con ciertos personajes y situaciones comienzan a desarrollar procesos de interpretación de sus propias historias de vida, generándose narraciones cada vez más conectadas con el sujeto que las relata. 
Narraciones que desde el relato de lo vivido e imaginado, hacen posible escuchar las voces acalladas de los niños y niñas desde una experiencia de diálogo abierto con ellos en las que emergen situaciones vividas, recordadas y olvidadas. En la lectura y la escritura de historias de vida, se reconoce un importante camino de trabajo pedagógico, que permite la formación de subjetividades reflexivas, en sintonía con el mundo de la experiencia, autocríticas, con conciencia de sí mismas y en movimiento constante.

Otro espacio importante para las discusiones, fueron los cine foros, a través de los cuales se propuso contar, leer, recrear y reconstruir historias como otra manera de acercarnos a la reflexión crítica de nuestras propias historias de vida, toda vez que nos permiten entrar en diálogo con nosotros mismos, con otros y con nuestro mundo. En este sentido, las películas también contribuyeron a desarrollar procesos de reflexión crítica en torno al papel de los niños y niñas en la sociedad, su lugar en la escuela, problemáticas de la infancia, y su actuación frente a situaciones de conflicto.

Empoderamiento a partir del lenguaje: Las películas provocaron en los niños y niñas distintas emociones, tristeza, sorpresa, miedo en algunos casos, revivieron recuerdos en ellos, se sintieron identificados con algunos personajes, lo cual permitió el desarrollo de conversaciones cargadas de un gran entusiasmo por hablar, participar de las discusiones, contar aspectos de sus propias vidas e imaginar situaciones alternativas. Evidenciándose en ellos, un gusto muy especial por las historias donde los niños y las niñas aparecen como protagonistas, donde son ellos los que cuentan las historias desde sí mismos, a partir de situaciones cotidianas.

En el desarrollo del tercer ciclo, 'Posicionamiento, o el reconocimiento de posibilidades de acción’, los procesos de reflexión crítica desarrollados durante el ciclo anterior, permitieron reconocer las posibilidades de acción de niños, niñas y maestros frente a las problemáticas presentadas en el contexto escolar y movilizarlas a través de prácticas educativas transformadoras de sentidos y significados.

Posicionamiento a partir de una acción creativa transformadora: Mediante la discusión colectiva de preguntas relacionadas con las temáticas: 'Un día en la escuela' y 'Un día con mi familia', se recrearon situaciones de la vida cotidiana a partir de las cuales los niños y niñas 
plantearon de manera creativa, rutas de acción transformadora para su vida escolar y familiar, desde las cuales los adultos (maestros) lograron reconocerlos como sujetos activos, participativos, reflexivos y críticos; logrando ver en ellos un gran potencial para actuar con otros niños, niñas y adultos en el desarrollo de propuestas colectivas y comunes desde las cuales se reconocen nuevas posibilidades de relación entre los adultos y los niños y niñas.

En tal sentido, las acciones propuestas por los niños y niñas se plantean como alternativas surgidas desde el pensamiento y el mundo infantil, acciones transformadoras de realidades en la medida en que emergen a partir de un cambio en la visión del mundo, que inicialmente era muy estrecha, determinista y mecanicista y que ahora se muestra mucho más abierta y crítica.

\section{Reflexiones finales}

El desarrollo de la propuesta permitió que los niños y niñas contribuyeran desde su formación, en la creación y dinamización de acciones tendientes a lograr una mayor participación, visibilización y reconocimiento de la infancia en el contexto escolar. En este sentido, la experiencia aporta nuevas miradas sobre la infancia, la escuela, la educación, lo político; desde las cuales se reconoce que en los niños y niñas la acción política es una acción creativa que rompe con la rutina escolar, instaurando nuevas formas de vinculación del sujeto con su contexto. Es una acción innovadora, en el sentido que posibilita nuevos comienzos, es inventiva y construye otros caminos.

Desde la perspectiva de la práctica pedagógica, el proceso desarrollado permite continuar cultivando el apasionamiento por la investigación en la perspectiva de formación y transformación de sujetos; logrando redescubrir intereses, gustos, experiencias que permiten la construcción de nuevos saberes y conocimientos desde miradas más amplias, dinámicas y flexibles sobre la infancia y la escuela. Por consiguiente, se reconoce en el recorrido del proceso, toda una experiencia de vida, movilizadora de pensamientos, actitudes, sentimientos, emociones, contradicciones y conflictos. Formativa en todos los sentidos, en lo personal, profesional y académico. 


\section{Referencias}

Arendt, H. (2001). ¿Qué es la política? Barcelona: Ediciones Paidós.

BÁrCenA, F. \& MÉLICH, J. C. (2000). La educación como acontecimiento ético.

Natalidad, narración y hospitalidad. Barcelona: Ediciones Paidós.

BÁrcena, F. (2006). Hannah Arendt: una filosofía de la natalidad. Barcelona:

Editorial Herder.

Carr, W \& Kemmis, S. (1988). Teoría Crítica de la Enseñanza. Barcelona: Martínez-Roca.

Cubides, H. (2006). Foucault y el sujeto político. Ética del cuidado de sí. Bogotá: Siglo del Hombre Editores, Universidad Central.

FreIRE, P. (1984). La importancia de leer y el proceso de liberación. México: Siglo Veintiuno Editores.

- (1997). La educación como práctica de la libertad. México: Siglo Veintiuno Editores.

. (1999). Política y Educación. México: Siglo Veintiuno Editores.

Martínez Boom, A. (2011). 'Unicef...dejad que los niños vengan a mí'.

Revista Educación y Pedagogía [23]. Universidad de Antioquia.

Narodowski, M. (1994). Infancia y poder. Argentina: Editorial Aique.

Ortega Valencia, P., Peñuela D., López, D. M. (2009). Sujetos y prácticas de las pedagogías críticas. Bogotá: Editorial El Búho. 